\title{
A novel strategy for identification of 47 pomegranate (Punica granatum) cultivars using RAPD markers
}

\author{
Y.P. Zhang ${ }^{1}$, H.H. Tan ${ }^{1,2}$, S.Y. Cao ${ }^{2}$, X.C. Wang ${ }^{1}$, G. Yang ${ }^{1}$ and J.G. Fang ${ }^{1}$ \\ ${ }^{1}$ College of Horticulture, Nanjing Agricultural University, Weigang, \\ Nanjing, P.R. China \\ ${ }^{2}$ Zhengzhou Institute of Pomology of Chinese Academy of Agricultural Sciences, \\ Zhengzhou, P.R. China \\ Corresponding author: J.G. Fang \\ E-mail: fanggg@njau.edu.cn
}

Genet. Mol. Res. 11 (3): 3032-3041 (2012)

Received January 26, 2011

Accepted May 6, 2012

Published May 30, 2012

DOI http://dx.doi.org/10.4238/2012.May.30.1

\begin{abstract}
DNA marker can be used for precise plant cultivar identification. However, DNA markers have often not been used effectively for the identification of plant cultivars due to a lack of an effective analysis strategy. We used a novel strategy for effective identification of plant individuals based on a new way of recording DNA fingerprints of the genotyped plants; a cultivar identification diagram can be manually generated and used as key reference information for quick identification of plant and/ or seed samples. Forty-seven pomegranate varieties popularly cultivated in various provinces of China were subjected to RAPD marker analysis. Using the cultivar identification diagram strategy, they were clearly separated by the fingerprints of 11 RAPD primers. The utility and accuracy of the cultivar identification diagram analysis results were confirmed by the identification of three randomly chosen groups of cultivars among the 47 varieties.
\end{abstract}

Key words: Pomegranate; Cultivar identification diagram; Verification; RAPD fingerprints 


\section{INTRODUCTION}

Pomegranate (Punica granatum L.) belongs to the Punicaceae family and is one of the oldest known edible fruits (Damania, 2005). The primary center of origin of pomegranates is believed to be in Iran, from where it has spread to other areas by humans (Simmonds, 1976; Levin, 1994). Pomegranate cultivation is common in China, Iran, India, Afghanistan, Mediterranean countries (Tunisia, Turkey, Egypt, Spain, and Morocco) and to some extent in the USA (California), Japan, and Russia (LaRue, 1980; Mars, 1994). In China, most pomegranate varieties popularly cultivated are landraces, and many of them were invariably given local or vernacular names by pomegranate growers. Scrutiny over the landrace names and their etymology, suggests that a given pomegranate landrace may be named differently in different regions and more than one landrace may share the same name thus causing some problems to breeders, commercial companies and farmers. This requires an accurate and rapid method for identification of pomegranate varieties, which is also important for cultivar-right-protection. Compared to the main fruit crops, pomegranate has not been given much attention from geneticists, cytogeneticists or molecular biologists. There have been reports on pomegranate cultivar identification and genetic analysis using molecular markers (Talebi-Baddaf et al., 2003; Sarkhosh et al., 2006; Masoud et al., 2008; Ebrahimi et al., 2010; Hasnaoui et al., 2010; Pirseyedi et al, 2010). However, the studies on effective identification of pomegranate cultivar are limited, since they utilized selected statistical techniques known as cluster analysis to study the banding pattern. These analyses were apparently not able to make an identification of the cultivars an easy and referable work, even though they could give the genetic diversity levels and separate individual plants in the final analysis results mostly shown as phylogenetic trees. Developing a strategy that can make the verification of pomegranate cultivars reliable, easy, and referable is necessary for nursery industry and growers as a tool for protecting plant patents and providing genetically uniform plants.

Compared to the morphological, physiological and agronomic traits used in pomegranate identification. Molecular markers are more advantageous as they are not affected by the environment, can provide a powerful tool for proper characterization of cultivars, are more informative, and do not need extensive observations of mature plants. Various DNA-based markers have been recently developed and used for studies in genetic diversity, fingerprinting and cultivar origin (Fang et al., 2005, 2006; Cheng and Huang, 2009; D'Onofrio et al., 2009; Elidemir and Uzun, 2009; Melgarejo et al., 2009; Papp et al., 2010). Among the several markers available, random amplified polymorphic DNA (RAPD) (William et al., 1990) is useful for cultivar analysis with major advantages in simplicity, efficiency, ease of operation, and non-requirement of any previous sequence information. If some optimization of the RAPD technique is achieved by choosing 11 nucleotide primers and strict screening PCR annealing temperature applied before the technique is employed in fingerprinting of plants, RAPD could be considered a preferred practical technique. To date, RAPD markers remain popular and have been used in the cultivar identification and genetic relationship analysis in a number of fruit tree species, such as apricot (Ercisli et al., 2009), pomegranate (Hasnaoui et al., 2010), cherry (Demirsoy et al., 2008), pistachio (Javanshah et al., 2007), and strawberry (Wang et al., 2007), etc. In practice, the powerful DNA markers available for plant identification have not made fruit crop variety identification an effective, recordable, and easy task as we measure the size of some plant parts using a ruler, which can be a rather awkward situation at times. The main bottleneck in such a situation is the analysis strategies of DNA fingerprints. Obviously, 
the popular analysis techniques for DNA banding patterns known as cluster analyses cannot render fruit crop cultivar or species separation an effective activity.

To address this setback, we developed and used a new strategy that can make identification of pomegranate cultivars practical, effective, recordable, and referable, in which a cultivar identification diagram (CID) was made manually with the polymorphic RAPD PCR bands generated from each primer shown. The presentation of the PCR band with the information of its size on the CID was an original invention that was first reported by our laboratory. The diagram generated, showing the separation of 47 pomegranate cultivars, can definitely be of service to the pomegranate industry particularly in China.

\section{MATERIAL AND METHODS}

\section{Plant materials}

The young leaves of 47 pomegranate cultivars were collected from the Institute of Pomology at the Chinese Academy of Agricultural Sciences, Zhengzhou. The name and origin of the cultivars are listed as in Table 1.

\section{Genomic DNA extraction}

Total genomic DNA of each genotype was extracted from young leaves using the modified cetyl trimethylammonium bromide (CTAB) method (Murray and Terompson, 1980; Bousquet et al., 1990). The extracted DNA was diluted to a final concentration of $30 \mathrm{ng} / \mu \mathrm{L}$ with $1 \mathrm{X}$ TE buffer and stored at $-20^{\circ} \mathrm{C}$ pending use.

\section{RAPD analysis}

In case of RAPD reactions, 11-mer primers were tested with several genotypes initially and only those primers resulting in clear unambiguous banding patterns with all genotypes tested were selected for use in genotyping. The use of 11-nucleotide primers (Table 2) was aimed at generating highly reproducible banding patterns based on our trial.

Reaction solutions consisted of $2.0 \mu \mathrm{L} 10 \mathrm{X}$ buffer, $1.2 \mu \mathrm{L} 25 \mathrm{mM} \mathrm{MgCl}_{2}, 1.6 \mu \mathrm{L} 2.5$ $\mathrm{mM}$ dNTP, $1.6 \mu \mathrm{L} 1.0 \mu \mathrm{M}$ primer, $0.1 \mu \mathrm{L} 5 \mathrm{U} / \mu \mathrm{L}$ rTaq Polymerase Dynazyme, and $1 \mu \mathrm{L}$ genomic DNA, making a total volume of $20 \mu \mathrm{L}$. Amplification reactions were performed based on the standard protocol of Williams et al. (1990) and the report of Demirsoy et al. (2008). The PCR was carried out in an Autorisierter Thermocycler (Eppendorf, Hamburg, Germany), programmed as follows: initial pre-denaturation stage for 5 min at $94^{\circ} \mathrm{C}$; then, 42 cycles each consisting of a denaturation stage for $30 \mathrm{~s}$; an annealing stage for $1 \mathrm{~min}$ at annealing temperature (Table 2), and an extension stage for $2 \mathrm{~min}$ at $72^{\circ} \mathrm{C}$. Amplification was terminated by a final extension of $72^{\circ} \mathrm{C}$ for $10 \mathrm{~min}$. After amplification, amplified DNA fragments were separated by gel electrophoresis on $1.3 \%$ agarose (w/v) (Figure 1) in $1 \mathrm{X}$ TAE (0.04 M Tris-acetate, $0.001 \mathrm{M}$ EDTA, pH 8.0) buffer at $100 \mathrm{~V}$. The gels were stained with $0.5 \mu \mathrm{g} / \mathrm{mL}$ ethidium bromide and visualized under ultraviolet light. Polymorphic bands among the cultivars were observed from photographs. In order to obtain reproducible, accurate and clear banding patterns, all amplifications were each repeated separately at least three times. 

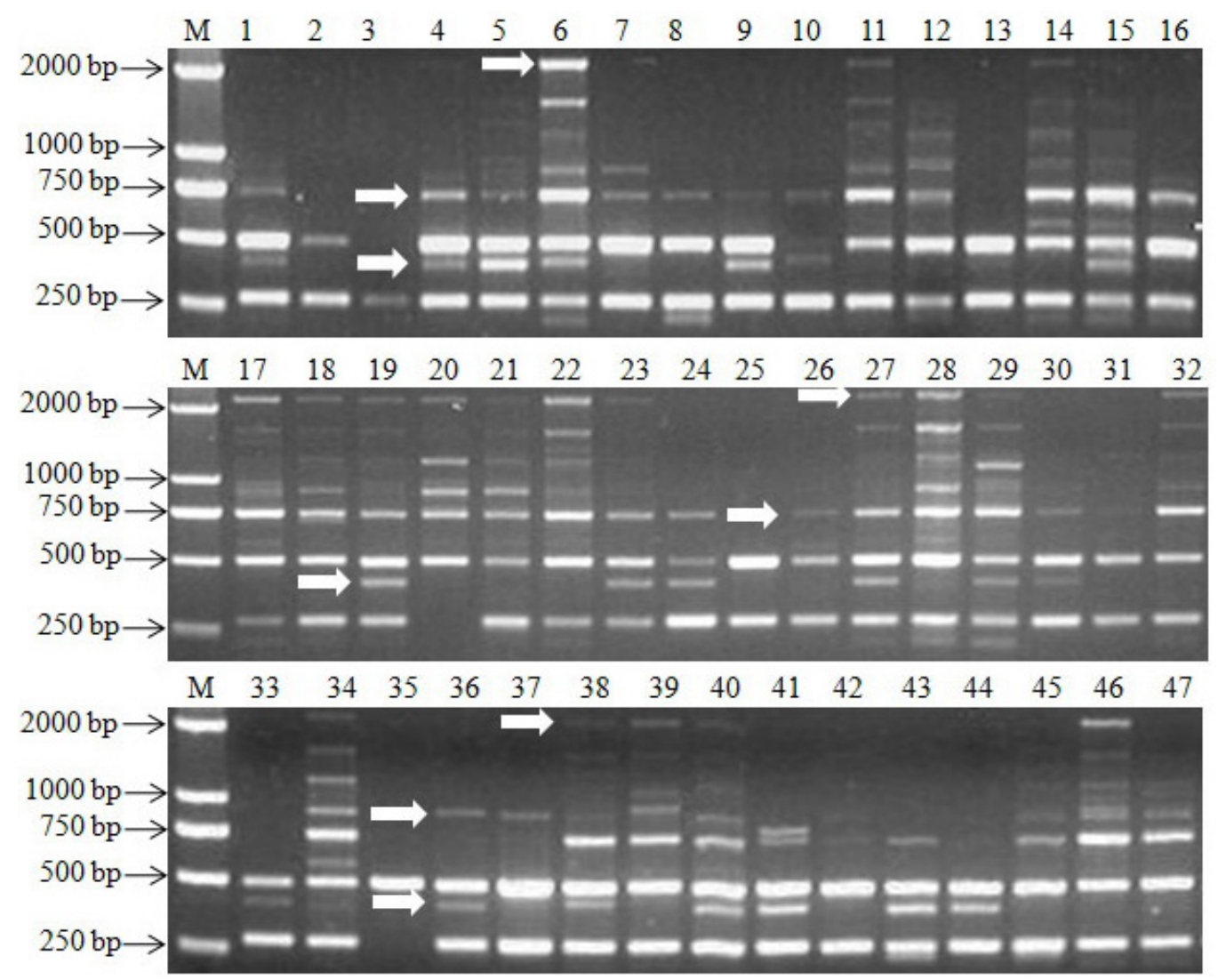

Figure 1. RAPD banding patterns of 47 pomegranate genotypes obtained with the primer Y57. White horizontal arrows indicate the polymorphic bands used for cultivar identification. Lane numbers correspond to the cultivar codes in Table 1. Lane $M=$ DNA size marker.

\section{Data analysis}

Only clear unambiguous bands in the photographic prints of gels were chosen and scored for cultivar identification. When some cultivars had specific band in the fingerprint generated from one primer, they could be separated singly, and those cultivars sharing the same banding pattern were separated into the same sub-group. In a similar manner, all the pomegranate cultivars were gradually and completely separated from each other with more primers employed.

\section{Test of utilization and workability of the diagram in cultivar identification}

Three groups of pomegranate cultivars, which were randomly chosen from the interand intra-groups, were used to verify the utilization and workability of the diagram showing the separation of the 47 cultivars. The three groups of cultivars were marked as "A", "B" and " $C$ ", and the corresponding primers to be used in separation of each group were easily located on the 
diagram. As these cultivars could be distinguished accurately and quickly based on the whole CID as anticipated, our results suggest that the strategy developed and employed in this study is scientific, workable, and effective, and could also be the optimal way to use molecular markers in the identification of other fruit crop cultivars and seed samples of field crops.

\section{RESULTS}

\section{Cultivar identification}

To establish a stable and optimistic RAPD system with high reproducibility, longer primers (11 nucleotides) were employed and the annealing temperatures for each primer were screened based on the quality and reproducibility of banding patterns. The primers were randomly screened from a stock of 60 11-mer primers, and once a suitable primer that could produce reproducible polymorphic bands was screened, it was utilized further in the identification of pomegranate cultivars. An example is the RAPD pattern, obtained with primer Y30 that was the first to be used in amplifying the 47 pomegranate cultivars in this study, where electrophoresis results show that primer Y30 generated uniform, and reproducible band patterns in 17 pomegranate cultivars assigned with the sample codes of $3,6,7,8,11,14,15,16,17,18,20,33,37,39,40,41$, and 47 as shown in Table 1. This group of cultivars was easily differentiated from the other 30 cultivars by presence or absence of a distinct $1800 \mathrm{bp}$ band, causing all the 47 cultivars to be separated into two groups. The second primer (Y6) used could then separate the two groups of cultivars earlier separated by primer Y30 into smaller groups or singly as "Huopao" and "Xindaian". This separation was done sequentially using another 9 primers (Table 2) screened and chosen to differentiate the pomegranate cultivars in the subgroups comprising of two or more cultivars. By primer Y57 (Figure 1), all the 47 cultivars

Table 1. Name and origin of the materials used in the experiment.

\begin{tabular}{rlllll}
\hline No. & Cultivar name & Origin & No. & Cultivar name & Origin \\
\hline 1 & Jiangshiliu & Shangxi & 25 & Moshiliu & Anhui\&Shandong \\
2 & Qingpiruanzi & Sichuan & 26 & Meizihong & $*$ \\
3 & Huopao & Yunnan & 27 & Piamanhongmei & Xinjiang \\
4 & Yushiliulhao & Henan & 28 & Qiaojianuomi & Yunnan \\
5 & Mudanhongsuanshiliu & Shandong & 29 & Juzimi & Shandong \\
6 & Dahongpao & Henan & 30 & Huilihongpi & Sichuan \\
7 & Heyinruanzi & Henan & 31 & Duanzhihong & Shandong \\
8 & Linxuan1hao & Shanxi & 32 & Dahongpao & Henan \\
9 & Henyinbopi & Henan & 33 & Yiruan & Shandong \\
10 & Huilihuangpi & Sichuan & 34 & Xindatian & Shanxi \\
11 & Tianhongdan & Shanxi & 35 & Heyinhuapi & Henan \\
12 & Yichenghong & Shandong & 36 & Wanchunhong & $*$ \\
13 & Gongyi & Henan & 37 & Zhuimeiren & Shanxi \\
14 & Dahongpitian & Hebei & 38 & Kaifeidahong1hao & Henan \\
15 & Manao & Anhui\&Shangdong & 39 & Huangsehuaguoshiliu & $*$ \\
16 & Dongshiliu & $*$ & 40 & Taishanghong & Shandong \\
17 & Tianlvzi & Yunnan & 41 & Linzalhao & Shanxi \\
18 & Yueliangbai & $*$ & 42 & Miandianjuxing & $*$ \\
19 & Xinjiangdahong & Xinjiang & 43 & Yushiliu & Shanxi \\
20 & Soft-seed Punica granatum from Tunisia & Tunisia & 44 & Yushi & Anhui \\
21 & Heyinsanbairuan & Henan & 45 & Sajinsi & Shanxi \\
22 & Mayatian & Shandong & 46 & Qingpihong & $*$ \\
23 & Yushiliu2hao & Henan & 47 & Chongkaiyueji & $*$ \\
24 & Yuejishiliu & Anhui & & & \\
\hline
\end{tabular}

*Indicates unknown origin of the cultivar. 
Table 2. Eleven primers used for the separation of the 47 pomegranate genotypes.

\begin{tabular}{llr}
\hline Primer & Nucleotide sequence $\left(5^{\prime}-3^{\prime}\right)$ & Annealing temperature $\left({ }^{\circ} \mathrm{C}\right)$ \\
\hline Y-4 & GTTTCGCTCCT & 44.8 \\
Y-6 & GTTTCGCTCCC & 44.8 \\
Y-9 & CTGCTGGGACA & 40.4 \\
Y-22 & GGACCCAACCT & 42.8 \\
Y-30 & GTGTGCCCCAC & 44.4 \\
Y-34 & AAGCCTCGTCT & 44.4 \\
Y-48 & ACGACCGACAC & 40.8 \\
Y-51 & TGGTGGCGTTA & 44.8 \\
Y-54 & TGGTGGCGTTC & 40.4 \\
Y-57 & ACCCCCGACTA & 44.8 \\
D-5 & GTCAGAGTCCT & 41.7 \\
\hline
\end{tabular}

were completely separated. The total set of 11 primers (Table 2 ) screened and utilized could identify all the 47 pomegranate cultivars as shown in Figure 2. What needs to be emphasized here is that only the clear polymorphic bands generated from each primer were used to differentiate the cultivars. Presentation of sizes and the presence/absence of polymorphic bands used in the CID as was done in Figure 2 can make the CID diagram very useful and referable in the practical pomegranate cultivar identification services. At each separation stage in the CID, the primer employed together with the polymorphic bands amplified by the primer can be considered as a scale-like scheme available for separation of the pomegranate cultivars. This therefore makes this CID strategy more useful in pomegranate cultivar identification than the currently popular cluster analysis.

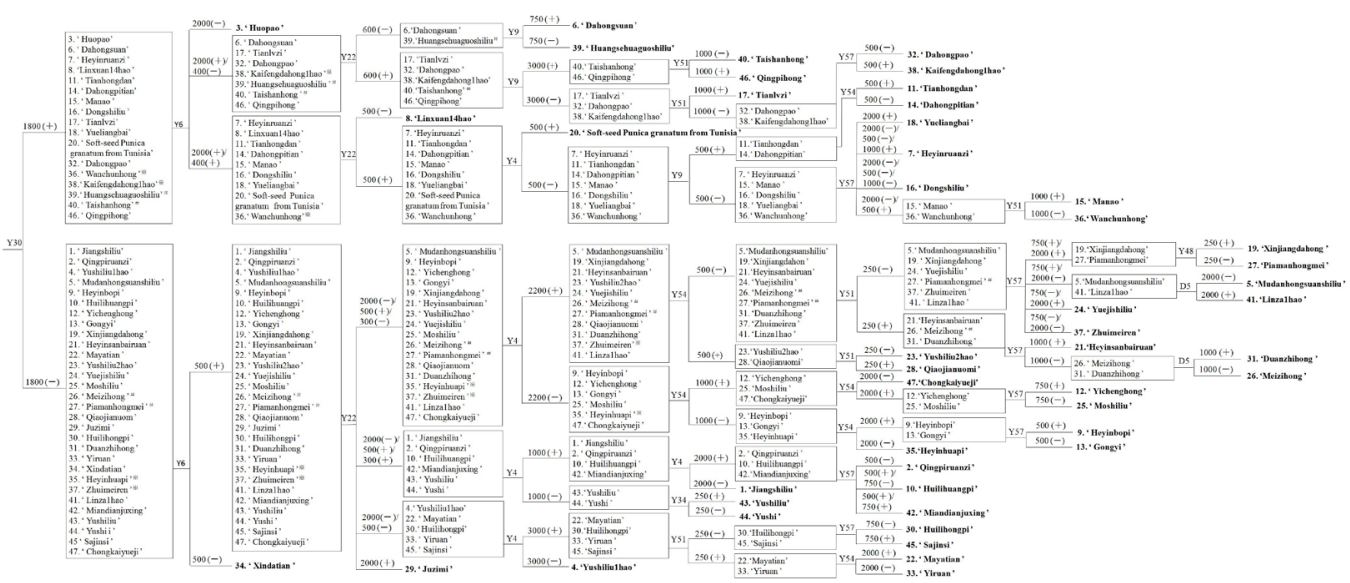

Figure 2. Cultivar identification diagram of the 47 pomegranate cultivars with the DNA fingerprints of 11 RAPD primers. Numbers above each horizontal line in the diagram $=$ the size of the polymorphic bands used to separate the cultivars following the line, reported in bp. $(+)=$ presence of the polymorphic band; $(-)=$ absence of polymorphic band; ' $*$ ' and ' $\#$ ' = cultivars selected to be used for the validation of the workability of the cultivar identification diagram. Cultivar names in bold are those that were separated.

\section{Test of utilization and workability of the cultivar identification diagram}

The most important aim of this study was not to show how to use RAPD marker to distinguish 47 pomegranate cultivars, since there have been numerous studies reported ear- 
lier that were mainly focused on the utilization of RAPD marker in identifying pomegranate and other plant cultivars via cluster analysis or direct fingerprinting of several plant samples. The most interesting and important purpose of this study was to generate a referable CID of pomegranates for future identification of these important cultivars popularly cultivated or used as valuable breeding parents in the pomegranate industry, which is also quite important to cultivar-right-protection. Therefore, it was necessary to verifiy the utilization, workability and efficiency of the diagram in pomegranate cultivar identification. To undertake this exercise, three groups of cultivars comprising "Heyinhuapi", "Wanchunhong", "Zuimeiren" and "Kaifengdahong1hao"; "Meizihong" and "Piamanhongmei"; "Huangsehuaguoshiliu" and "Taishanghong", which came from the inter- and intra-groups or subgroups in the CID, were randomly chosen and used for the verification. Based on the location of these cultivars in CID, it was easy to locate the primers to be used to separate these three groups of cultivars where the primers chosen were Y30, Y4, Y6, Y51, and Y22. PCR results exhibited the banding patterns as anticipated whereby all cultivars in these three groups could be disjoined and separated using specific polymorphic bands marked in the CID. The four pomegranate cultivars in the first group could be separated by the PCR using primers Y30, Y4, and Y6 (Figure 3A). The 1800-bp band generated by Y30 could first separate them into two groups, from which the group comprised of "Zuimeiren" and "Heyinhuapi" could be further disjoined with the primer Y4 by the 2200-bp band while the other group of "Wanchunhong" and "Kaifengdahong1hao" were divided by primer Y6 with the band of about 400 bp in size. The group of "Meizihong" and "Piamanhongmei" could be separated by the 250-bp long polymorphic band derived from primer Y51 (Figure 3B). The band of approximately $600 \mathrm{bp}$ in length from primer Y22 disjoined the group of "Huangsehuaguoshiliu" and "Taishanghong" (Figure 3C).

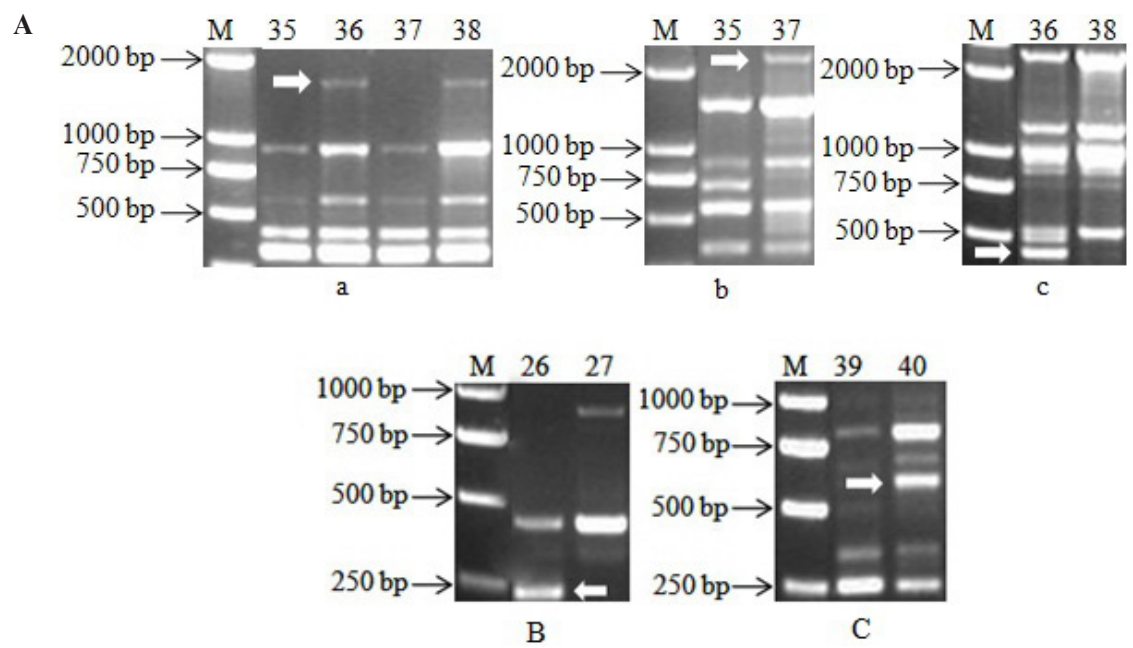

Figure 3. Verification result of cultivars selected randomly by the corresponding primers. White horizontal arrows indicate the anticipated polymorphic bands. Lane numbers correspond to the code in Table 1. Lane $M=$ DNA size marker. A. Fingerprint obtained with three primers used to separate the first group of cultivars selected (marked with " $¥ "$ in Figure 2). a. b. and c. were obtained with the primers Y30, Y4, and Y6, respectively. B. Fingerprint obtained with the primer Y51 used to separate the group 'Meizihong' and 'Piamanhongmei' (marked with "\#" in Figure 2). C. DNA fingerprint obtained with the primer Y22 used to separate the group 'Huangsehuaguoshiliu' and 'Taishanghong' (marked with “\#” in Figure 2). 
This validation of separation of the randomly chosen groups of cultivars could show that this pomegranate cultivar identification strategy was in fact practicable, workable, effective, and referable, and could be of vital service to the pomegranate industry. It is worth mentioning that the data of cultivar separation from the CID can also be generated into a database for future use in silico.

\section{DISCUSSION}

DNA markers are a category of powerful techniques used to identify plant cultivars and species. Although several generations of DNA markers have been developed and used in cultivar identification, genetic analysis, and thousands of related papers have been published, it does not imply that they have been easily and widely used in genotyping. In fact, the desire to use DNA markers to identify plant varieties effective and easily is normally hindered by a dearth of practical ways of harnessing this resource. To date, no effective approach had been developed to use DNA markers easily in cultivar identification apart from the use of phylogenetic trees or some fingerprints of several cultivars. This new approach developed in this study to use DNA markers in distinguishing cultivars is in fact more effective and practical, as well as being less costly. It can be quickly operated and referable in addition to other benefits. This strategy can fully utilize the power of DNA markers in plant cultivar identification, in which clear polymorphic bands from each primer tried can be used to gradually distinguish the individual samples and chart the identification results informatively and clearly. Although the method does not accurately reflect the genetic relationship of the plant cultivars, theoretically, the first cultivar to be separated out will definitely have the farthest genetic distance between it and the others while those identified and separated later, might be closer genetically. This method can be of great help in plant cultivar identification for cultivar-right-protection, cultivar identification, and early identification in the nursery plant industry.

China is a leading agricultural country in the world and has a great amount of plant genetic resources, which makes the differentiation of plant samples an important task. Pomegranate is an ancient horticultural crop and has been cultivated on a large scale in most provinces of China in recent years. Despite its importance, little work has been reported on effective cultivar identification and genetic diversity of pomegranate. At present, the phenomenon where a name might be used for various pomegranate cultivars or a pomegranate cultivar has different names in different production regions is quite common in China. Development of the pomegranate industry requires related enhancement of pomegranate research and production. Therefore, scientific identification of pomegranate cultivars and germplasm resources is essential, since it can be of great help to genetic resource conservation and utilization as well as plant variety protection.

In this study, 11 RAPD primers were sufficient to distinguish all the 47 pomegranate cultivars with the employment of the CID strategy. This strategy is very convenient and fast in practice. Although a single RAPD primer cannot distinguish all pomegranate cultivars at the same time, this method can make the advantages of DNA markers really workable in plant identification, and shows a substantial increase in efficacy over previous studies that were mainly focused on cluster analysis. This study provides new evidence regarding the rapid identification of pomegranate cultivars. The informative CID diagram (Figure 2) of the pomegranate cultivars can indicate the primers to use in separating particular pomegranate cultivars. 
Basically, any two cultivars can be identified with one RAPD primer. If more cultivars need to be separated, the primers that are required can also be easily located from the CID. In practice, if more new pomegranate cultivars are released in production, the set of 11 primers can be used to run the DNA samples of the new cultivars and the PCR banding patterns can show where to position the new cultivars in the CID. If they cannot be disjoined with the 47 ones by the 11 primers, new primers can be used to separate and position them on the CID. Since this strategy does not involve much work, it can be used to generate a larger CID of pomegranate cultivars, which is definitely significant in the pomegranate industry.

This is the first report, to our knowledge, that assesses the use of RAPD primers in complete identification of pomegranate cultivars. Verification of workability and accuracy of the pomegranate CID satisfactorily gave the anticipated end results. This could also suggest the utility and reliability of RAPD marker in plant identification. Therefore, this study could prompt new work for effective application of DNA markers even in identification of other plant and seed samples, which are important in plant genetic germplasm conservation, cultivar-right-protection, provision of genetically uniform seedlings in production, and in the seed industry. This CID plant cultivar identification has some advantages where fewer primers can be effectively used, all cultivars included can be separated now and in the future by the corresponding primers easily found on the diagram, CID information can be transferred to e have previously initiated similar work on most important fruit crop cultivars cultivated in China for service to cultivar-right-protection, nursery plant industry, and genetic resource conservation. We further believe that this new method can be used to draw the CIDs for various organisms and species, and the CIDs drawn can work in a similar manner to the periodic table of chemical elements, providing us with the information needed to separate the cultivars planned.

\section{ACKNOWLEDGMENTS}

Research supported by the NCET Program of China (grant \#NCET-08-0796) and the Fundamental Research Funds for the Central Universities (\#KYJ200909).

\section{REFERENCES}

Bousquet J, Simon L and Lalonde M (1990). DNA amplification from vegetative and sexual tissues of tree using polymerase chain reaction. Can. J. Forest Res. 20: 254-257.

Cheng Z and Huang H (2009). SSR fingerprinting Chinese peach cultivars and landraces (Prunus persica) and analysis of their genetic relationships. Sci. Horticult. 120: 188-193.

Damania AB (2005). The Pomegranate: Its Origin, Folklore, and Efficacious Medicinal Properties. In: Agriculture Heritage of Asia - Proceedings of the International Conference (Nene YL, ed.). Asian Agri History Foundation, Secunderabad, 175-183.

Demirsoy L, Demir T, Demirsoy H, Okumus A, et al. (2008). Identification of some sweet cherry cultivars grown in Amasya by RAPD markers. Acta Horticult. 795: 147-152.

D’Onofrio C, Lorenzis G, Giordani T, Natali L, et al. (2009). Retrotransposon-based molecular markers in grapevine species and cultivars identification and phylogenetic analysis. Acta Horticult. 827: 45-52.

Ebrahimi S, Sayed-Tabatabaei BE and Sharifnabi B (2010). Microsatellite isolation and characterization in pomegranate (Punica granatum L.). Iranian J. Biotechnol. 8: 156-163.

Elidemir AY and Uzun I (2009). Assessment of genetic diversity of some important grape cultivars, rootstocks, and wild grapes in Turkey using RAPD markers. Acta Horticult. 827: 275-278.

Ercisli S, Agar G, Yildirim N, Esitken A, et al. (2009). Identification of apricot cultivars in Turkey (Prunus armeniaca L.) using RAPD markers. Romanian Biotechnol. Letter 14: 4582-4588. 
Fang J, Qiao Y, Zhang Z and Chao CT (2005). Genotyping fruiting-mei (Prunus mume Sieb. Et Zucc) cultivars using AFLP. Hort. Sci. 40: 325-328.

Fang J, Twito T, Zhang Z and Chao CT (2006). Genetic relationships among fruiting-mei (Prunus mume Sieb. et Zucc.) cultivars evaluated with AFLP and SNP markers. Genome 49: 1256-1264.

Hasnaoui N, Messaoud M, Jemni C and Mokhtar T (2010). Molecular polymorphisms in Tunisian pomegranate (Punica granatum L.) as revealed by RAPD fingerprints. Diversity 2: 107-114.

Javanshah A, Tajabadipour A and Mirzaei S (2007). Identification of a new phenotype (Siah Barg) of pistachio (Pistacia vera L.) with shiny-blackish green leaves using RAPD assay. Int. J. Agri. Biol. 9: 307-310.

LaRue JH (1980). Growing Pomegranates in California. Farm Advisor, Tulare County from: DANR Leaflet, California.

Levin GM (1994). Pomegranate (Punica granatum L.) plant genetic resources in Turkmenistan. Plant Genet. Resour. Newslett. 97: 31-36.

Mars M (1994). La Culture du Grenadier (Punica granatum L.) et du Figuier (Ficus carica L.) en Tunisia. First Meeting CIHEAM Coop. Res. Network on Underutilized. Fruit Trees, Zaragoza.

Masoud S, Saneghi A, Shahreiyari ZH, Noormohammadi Z, et al. (2008). RAPD and cytogenetic study of some pomegranate (Punica granatum L.) cultivars. Caryologia 61: 68-73.

Melgarejo P, Martínez JJ, Fca H, Martínez R, et al. (2009). Cultivar identification using 18S-28S rDNA intergenic spacerRFLP in pomegranate (Punica granatum L.). Sci. Horticult. 120: 500-503.

Murray GC and Thompson WF (1980). Rapid isolation of high molecular weight DNA. Nucl. Acids Res. 8: 4321- 4325.

Papp N, Szilvássy B, Abrankó L, Szabó T, et al. (2010). Main quality attributes and antioxidants in Hungarian sour cherries: identification of genotypes with enhanced functional properties. Int. J. Food Sci Tech. 45: 395-402.

Pirseyedi SM, Valizadehghan S, Mardi M, Ghaffari MR, et al. (2010). Isolation and characterization of novel microsatellite markers in pomegranate (Punica granatum L.). Int. J. Mol. Sci. 11: 2010-2016.

Sarkhosh A, Zamani Z, Fatahi R and Ebadi A (2006). RAPD markers reveal polymorphism among some Iranian pomegranate (Punica granatum L.) genotypes. Sci. Horticult. 111: 24-29.

Simmonds NW (1976). Evolution of Crop Plants. Longman, London.

Talebi-Baddaf M, Sharifineia B and Bahar M (2003). Analys is of Genetic Diversity in Pomegranate Cultivars of Iran, Using Random Amplified Polymorphic DNA (RAPD) Markers. Proceeding of the Third National Congress of Biotechnology. Iran, 2: 343-345.

Wang ZG, Zhang ZH, Li H, Gao XY, et al. (2007). Identification of strawberry cultivars by RAPD and SCAR markers. Acta Horticult. Sin. 34: 591-596.

Williams JG, Kubelik AR, Livak KJ, Rafalski JA, et al. (1990). DNA polymorphisms amplified by arbitrary primers are useful as genetic markers. Nucleic Acids Res. 18: 6531-6535. 